\title{
Study on the Improvement of LGDM Based on the Conflict and Risk Perception
}

\author{
Xuanhua Xu, Zhipeng Ma, Yanfen Yu, Yania Huang, Zhao Yang \\ School of Business, Central South University, Changsha 410083, China \\ 基于冲突和风险感知的重大突发事件大群体应 \\ 急决策品质提升研究 \\ 徐选华, 马志鹏, 余艳粉, 黄燕霞, 杨昭 \\ 中南大学商学院, 长沙 410083, 中国
}

Abstract

The logical connection and impact of group

事件大群体决策品质研究的前沿问题。本文以 conflict and risk perception on the quality improvement of 1 emergency LGDM are the frontier issues in the study of the quality of emergency LGDM. Based on risk decision theory and conflict theory, this paper studies the mechanism of group conflict and risk perception affecting the quality of emergency LGDM by constructing a structural equation model containing mediator variables. The empirical results show that task conflict has a positive impact on the quality of emergency decision making through risk-awareness behavior, and the mediating role of risk perception in relationship conflict and emergency LGDM is not significant. The conclusions of this study reveal the internal mechanism of the external factors in the research of the mechanism of the external factors, and provide theoretical and practical values for exploring the improvement and improvement of the quality of emergency LGDM.

Keywords: group conflict; risk perception; emergency decision making; decision quality

摘要

群体冲突与风险感知对大群体应急决策 风险决策理论和冲突理论为基础, 通过构建一 个包含中介变量的结构方程模型, 对群体冲突、 风险感知影响大群体应急决策品质的机制进 行研究。实证结果表明, 任务冲突通过风险感 知行为对应急决策品质产生积极影响, 而风险 感知在关系冲突与应急决策品质之间的中介 作用不显著。本研究的结论揭示了内在层面因 素对外在研究领域的作用机理, 为探索大群体 应急决策品质改善与提升提供了理论与实际 价值。

关键词: 群体冲突; 风险感知; 应急决策; 决 策品质

1. 引言

近年来, 我国重特大突发事件的数量呈明 显上升趋势, 涉及范围明显扩大, 事件的复杂 性和应对难度显著加大, 处置紧迫性亦不断提 高, 给我国经济和人民生命财产造成重大损失。 由此, 对专家群体的规模和领域分布都提出了 更高的要求。然而, 尽管大群体应急决策与处 置已有大量方法和算例证明 [12], 但在当前的 应急处置环境下却难以达成高质量的群体决 策。主要由于大群体决策专家领域知识分散, 行业分布广泛的专家群体易形成偏好冲突, 难 以在短时间内达成高度共识的决策内容, 导致 大群体决策的整体效率和价值较低。鉴于此, 大群体应急决策亟需借助科学有效的管理方 品质提升的逻辑联系及影响路径, 是重大突发 
式, 推动高质量应急决策的制定和实施, 进而 实现有效的应急处置。

为弥补上述不足, 本文基于冲突理论和风 险决策理论划分了冲突的构成维度 [5], 考虑了 群体冲突与应急决策品质提升之间的关系, 以 及风险感知对上述路径的中介作用, 以期探明 大群体决策专家冲突与应急决策品质提升之 间的“黑箱”。相较于已有研究, 本文提出了一 个更全面、层次多样的研究框架, 系统审视了 大群体专家冲突与应急决策品质之间的关系, 揭示了风险感知在两者中的特殊作用。研究结 论对于揭示大群体冲突内涵, 丰富应急环境下 的风险决策理论, 以及完善突发事件处置流程 与政策制定等方面, 具有重要的理论意义和实 际意义。

\section{2. 理论基础与研究假设}

\section{1 群体冲突与应急决策品质}

在组织行为学的研究中, 冲突被定义为组 织中个体间的观点差异或者不相容 [1]。根据 Amason 和 Jehn 的研究, 可进一步将冲突划分 为任务冲突和关系冲突 [2][3]。前者是指冲突 目的与群体任务相一致的功能性冲突, 即注重 如何更好的完成共同目标, 补足认知上的差异。 而后者则是注重情绪上宣泄与争辩, 即非功能 性的情感表达。

一方面, Ring 和 Van 对决策团队的研究, 实证了任务冲突对于提升决策质量的积极作 用 [6]。同时, 不同意见的交流和碰撞亦有利于 对决策方案形成批判性评价[3], 降低了群体思 维的风险; 另一方面, Simons 和 Peterson 认为 关系冲突对团队决策绩效有负向影响 [7]。关系 冲突使群体关注的焦点偏离任务本身, 限制了 团队决策效率和信息处理能力。同时, 关系冲 突增加了群体内部不和谐因素和负面情绪, 导 致决策品质低下甚至极端决策出现[1]。基于此, 本文提出假设:

H1a 决策专家间的任务冲突对群体决策 品质具有正相关关系

H1b 决策专家间的关系冲突对群体决策 品质具有负相关关系

\section{2 群体冲突与风险感知}

既有研究将风险感知描述为人们在特定 情境下, 对待不确定事件的态度和直觉判断, 具有高度的变化性和情境依赖性[4]。既包含人 们在面对风险时做出的直观判断, 也包括经过 理性和复杂分析后得出的主观认识。

一方面, 情境启发理论表明, 风险决策的 过程中, 决策者往往需要借助特定的意象或情 境来激活相应的风险感知。Slovic (2002) 的 研究同样强调了直觉和经验, 而非复杂的理论 分析, 在个体风险判断中的重要作用 $[10]$ 。任 务冲突拓展了该种互补性意象和情境的传播 和获取渠道, 使得专家的风险直觉感知更加全 面; 另一方面, 关系冲突使得个体之间的信息 交流受阻, 难于产生正面的情绪激励。Peter （2004）提出了风险感知情绪模型, 认为情绪 反应的强烈程度, 是影响个体风险感知差异的 关键因素[18]。由于情绪本身具有行为导向性, 个体负面情绪会加重群体间的不信任和猜疑, 使得风险感知和决策行为夹杂了更多的个人 情感因素。基于此, 本文提出假设:

H2a 决策专家间的任务冲突对风险感知 具有正相关关系

H2b 决策专家间的关系冲突对风险感知 具有负相关关系

\section{3 风险感知与应急决策品质}

风险决策理论认为, 人们的决策是建立在 其对风险的评估与判断基础之上，而风险评估 和风险判断又受认知层面的影响 [8]。Dash （2010）研究指出, 机会感知、风险感知和风 险偏好均会显著影响最终决策[10], 在应急决 策过程中, 决策专家致力于风险性因素的消减 和规避, 有利于最大限度降低突发事件风险水 平、提高事态可控性和结果可预测性。Williams （2007）的研究进一步将风险感知视作决策过 程中固有的一部分 [13], 认为参照风险感知的 决策行为更有利于形成富有针对性的策略。基 于此, 本文提出假设:

H3 决策专家的风险感知对群体决策品 质具有正相关关系 


\section{4 风险感知的中介作用}

仅从专家群体冲突的视角切入应急决策 品质的研究, 缺乏了对决策者心理及微观视角 的探索过程, 难以形成全面客观的结果致因及 作用逻辑[9]。由此, 本文结合由群体冲突引发 的专家认知和偏好等心理行为变化, 将风险感 知作为大群体冲突与应急决策品质之间的中 介变量。风险感知的多元化作用弥补了决策专 家“复杂偏好异质性”与群体共同目标之间的 矛盾, 承接了群体“预期目标”与“方案修正、 达成”之间的关系。一方面, 频繁的任务冲突 填补了专家对于事件的认知空白, 进而影响群 体的偏好选择与判断, 最终左右决策策略; 另 一方面, Peter (1996) 提出风险感知模型[10], 认为波动的情绪会降低群体对于风险因素的 敏感程度, 导致消极或惩罚性的后果, 最终影 响决策品质。基于此, 本文提出假设:

H4a 风险感知在决策专家任务冲突对群 体决策品质的影响路径中起到中介作用, 即任 务冲突通过改变决策专家风险感知影响群体 决策品质

H4b 风险感知在决策专家关系冲突对群 体决策品质的影响路径中起到中介作用, 即关 系冲突通过改变决策专家风险感知影响群体 决策品质根据上述分析, 本文提出大群体决策 者冲突行为对应急决策绩效的影响机制, 认为 群体决策者的任务冲突和情绪冲突会通过风 险感知的中介作用影响最终的应急决策品质。 本文的概念模型如图 1 所示。

\section{3. 假设检验与决策品质提升启示}

本研究以天津港“8.12”特别重大火灾爆 炸事件等案例的专家决策群体为调查对象。主 要原因是基于我国特大突发事件的背景, 上述
决策群体具有明显的大规模动态性和复杂异 质性, 导致决策过程中的专家间差异和冲突更 为明显, 对潜在和未知风险感知识别的要求更 高, 更有意愿通过合作形成高品质的应急决策 [11]。

\section{1 信度与效度检验}

表 1 为各量表的信度和效度信息。可知, 各个量表的解释均方差都大于 $55 \%$, Cronbach's $\alpha$ 值均大于 0.8 , 表明研究使用的量 表均有良好的内部一致性信度。各观测变量的 $\mathrm{KMO}$ 值均大于 0.7 , 表明变量内部题项之间具 有共同因素。

表 1 量表的信度与效度（N=423）

\begin{tabular}{ccccc}
\hline 测量变量 & 题项 & Cronbach & KMO 值 & 解释均方 \\
\hline 任务冲突 & 4 & 0.819 & 0.790 & $61.28 \%$ \\
关系冲突 & 3 & 0.838 & 0.711 & $65.66 \%$ \\
风险感知 & 6 & 0.865 & 0.870 & $59.74 \%$ \\
应急决策品质 & 9 & 0.835 & 0.912 & $57.07 \%$ \\
\hline
\end{tabular}

\section{2 描述性统计分析}

由于应急决策专家的共同意愿是形成应 对方案, 进而控制风险事件并降低危害, 故借 鉴Jehn 和 Simon 对此类研究的控制变量设计, 用年龄、受教育程度、从业领域和职业层次作 为控制变量。由表 2 可知各研究变量的均值、 标准差及相关系数。可知, 各变量之间具有一 定的相关性, 但相关系数不高, 说明变量之间 不存在严重的多重共线性问题。

\section{3 假设检验}

主效应及简单假设检验如表 3 。中介效果 的检验参考 MacKinnon(2007)的方法[19], 利 用 Bootstrap 技术重新估计间接 效果的标准 误及信赖区间。通过表 4 可知, 任务冲突 Bootstrap 检验方法的信赖区间均未包含 0 , 且

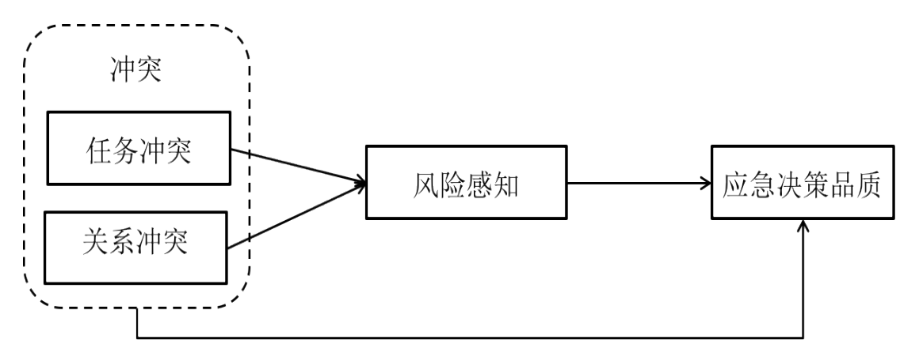

图 1 研究的概念模型图 
表 2 量表的描述性统计分析（ $\mathrm{N}=423 ）$

\begin{tabular}{ccccccccc}
\hline 变量 & 1 & 2 & 3 & 4 & 5 & 6 & 7 & 8 \\
\hline 1.年龄 & $\mathbf{1}$ & & & & & & & \\
2.教育程度 & 0.10 & $\mathbf{1}$ & & & & & & \\
3.从业领域 & 0.02 & -0.0 & $\mathbf{1}$ & & & & & \\
4.职业层次 & 0.09 & 0.08 & 0.00 & $\mathbf{1}$ & & & & \\
5.任务冲突 & 0.03 & 0.11 & 0.09 & 0.02 & $\mathbf{1}$ & & & \\
6.关系冲突 & 0.03 & 0.08 & 0.07 & 0.06 & -0.23 & $\mathbf{1}$ & & \\
7.风险感知 & 0.00 & 0.07 & 0.03 & 0.05 & 0.413 & -0.21 & $\mathbf{1}$ & \\
8.应急决策 & 0.08 & 0.00 & 0.05 & 0.07 & 0.391 & -0.29 & 0.472 & $\mathbf{1}$ \\
Average & 2.44 & 2.36 & 1.97 & 2.29 & 5.775 & 5.761 & 6.001 & 6.21 \\
SD & 1.25 & 0.80 & 1.12 & 0.61 & 0.529 & 0.542 & 0.566 & 0.43 \\
\hline
\end{tabular}

表 3 主效应 Bootstrap 分析结果 $(\mathrm{N}=423)$

\begin{tabular}{ccccc}
\hline 路径 & 标准化路径系数 & $\mathrm{T}$ 值 & $\mathrm{P}$ & 结论 \\
\hline 任务冲突->应急决策品质 & 0.480 & 7.140 & $* * *$ & 支持 \\
关系冲突->应急决策品质 & -0.400 & 5.592 & $* * *$ & 支持 \\
任务冲突-> 风险感知 & 0.350 & 4.940 & $* * *$ & 支持 \\
关系冲突-> 风险感知 & 0.078 & 0.798 & - & 不支 \\
风险感知->应急决策品质 & 0.320 & 4.517 & $* * *$ & 支持 \\
卡方 $\quad$ 448.472 自由度 df & 348 & 自由卡 & 1.289 \\
Goodness of Fit Index (GFI) & & 0.920 \\
Adjusted Goodness of Fit Index (AGFI) & & 0.900 \\
Root Mean Square Error of Approximation (RESEA) & 0.031 \\
Comparative Fit Index (CFI) & & 0.981 \\
Incremental Fit Index (IFI) & & 0.981 \\
Tucker-Lewis Index (TLI) & & 0.978 \\
\hline
\end{tabular}

表 4 任务冲突 Bootstrap 分析结果 $(\mathrm{N}=423)$

\begin{tabular}{|c|c|c|c|c|c|c|c|}
\hline \multirow{3}{*}{ 变数 } & \multirow{3}{*}{ 点估计 } & \multicolumn{2}{|c|}{ 系数相乘积 } & \multicolumn{4}{|c|}{ Bootstrap } \\
\hline & & \multicolumn{2}{|c|}{ Product of Coefficients } & \multicolumn{2}{|c|}{ Bias-Corrected $(95 \% \mathrm{CI})$} & \multicolumn{2}{|c|}{ Percentile $(95 \% \mathrm{CI})$} \\
\hline & & SE & Z & Lower & Upper & Lower & Upper \\
\hline 任务冲突->应急决策品质 & 0.339 & 0.091 & $\begin{array}{c}\text { Total Effects } \\
3.725\end{array}$ & 0.160 & 0.508 & 0.157 & 0.506 \\
\hline 任务冲突->应急决策品质 & 0.065 & 0.029 & $\begin{array}{c}\text { Indirect } \\
2.241\end{array}$ & 0.021 & 0.138 & 0.014 & 0.128 \\
\hline 任务冲突->应急决策品质 & 0.275 & 0.086 & $\begin{array}{c}\text { Direct Effects } \\
3.198\end{array}$ & 0.094 & 0.435 & 0.094 & 0.435 \\
\hline
\end{tabular}

$\mathrm{Z}$ 值大于 1.96 , 说明风险感知在任务冲突与应 急决策品质之间的总效应和间接效应均存在, 直接效果存在且显著, 即风险感知的中介作用 为部分中介。同理可知, 表 5 中风险感知在关 系冲突与应急决策品质之间的中介效果不显 著。

\section{4 决策品质提升启示}

实证结果表明, 群体冲突、风险感知行为与大 群体应急决策品质之间的存在显著相关关系。 一方面, 本文证实了群体冲突与应急决策品质 提升之间的显著关系, 其中任务冲突有利于形 成高品质应急决策, 而群体内部关系冲突则不 利于形成高品质的应急决策。可以解读为任务 
表 5 关系冲突 Bootstrap 分析结果 $(\mathrm{N}=423)$

\begin{tabular}{|c|c|c|c|c|c|c|c|}
\hline \multirow{3}{*}{ 变数 } & \multirow{3}{*}{ 点估计 } & \multicolumn{2}{|c|}{ 系数相乘积 } & \multicolumn{4}{|c|}{ Bootstrap } \\
\hline & & \multicolumn{2}{|c|}{ Product of Coefficients } & \multicolumn{2}{|c|}{ Bias-Corrected $(95 \% \mathrm{CI})$} & \multicolumn{2}{|c|}{ Percentile $(95 \% \mathrm{CI}$} \\
\hline & & SE & Z & Lower & Upper & Lower & Upper \\
\hline 关系冲突->应急决策品质 & 0.177 & 0.101 & $\begin{array}{c}\text { Total Effects } \\
1.752\end{array}$ & 0.114 & 0.262 & 0.088 & 0.271 \\
\hline 关系冲突->应急决策品质 & 0.031 & 0.020 & $\begin{array}{c}\text { Indirect } \\
1.550\end{array}$ & -0.079 & 0.104 & -0.113 & 0.095 \\
\hline 关系冲突->应急决策品质 & 0.124 & 0.093 & $\begin{array}{c}\text { Direct Effects } \\
3.198\end{array}$ & 0.063 & 0,179 & 0.044 & 0.181 \\
\hline
\end{tabular}

冲突的广泛存在促进了组织中互补性知识的 交换, 提升了团队成员运用相关经验方法处置 特定事件的能力, 进而有效改善应急决策品质。 而关系冲突则降低了决策专家的经验分享意 愿, 阻碍了群体中的信息交流和知识传递, 不 利于形成事件的多维认知, 由此降低了应急决 策品质的有效性。

另一方面, 本文结论进一步探明了风险感 知的中介作用, 在应急决策品质提升中的关键 作用, 对于解开群体冲突与应急决策品质之间 的作用 “黑箱” 具有重要价值。此前学者的研究 多从信息沟通和个体情感等方面设置中介路 径, 已有的成熟中介变量有群体信息交互[14], 群体凝聚力[15]。和团队心智模型[16][17]。本 研究进一步完善了“群体冲突—群体绩效”路 径中风险感知的中介效应, 可以解读为风险感 知的功能性作用进一步发挥了由任务冲突带 来的丰富的信息价值, 更为细致地阐述了应急 决策品质提升的内在逻辑。

\section{4. 研究结论与展望}

以上研究启示对于突发事件应急处置具 有一定的应用价值: 其一, 应当注重群体专家 的规模和领域分布, 并通过一定的方式激励专 家群体开展知识经验交流, 填补群体内部的知 识空缺; 其二, 应及时控制和疏导组织内部的 负面情绪, 避免组织内部的不和谐或关系紧张, 防止关系冲突的产生和升级, 导致的应急决策 品质的质量和效率降低。综上所述, 本文的研 究结论基于团队层面, 为风险决策理论和冲突 理论在决策中的拓展提供了新的实证依据, 为
大群体应急决策品质提升提供了新的思路方 法。

尽管本研究取得了一些有价值的论断, 但 仍然需要完善。后期可以对群体规模及领域细 分等控制变量进行完善和细化, 加大实验样本 的数量进行对比分析, 进一步探索群体冲突对 应急决策品质提升的影响, 以期获得更具说服 力和适用性的结论。

\section{Acknowledgements}

This study was supported by National Social Science Foundation of China(No.71790615 and No. 71790615)

\section{致谢}

本研究得到国家自然科学基金项目 (71671189); 国家自然科学基金重点项目 （71790615）的资助。

\section{参考文献}

[1] 郎淳刚, 席酉民, 郭士伊. 团队内冲突对 团队决策质量和满意度影响的实证研究. 中国工商管理研究前沿,2008, 19(1): $10-15$.

[2] Amason A C. Distinguishing the effects of functional and dysfunctional conflict on strategic decision making: resolving a paradox for top management teams. Academy of Management Journal, 1996, 39(1):123-148.

[3] Jehn K A. A Multimethod examination of the benefits and detriments of intragroup 
conflict. Administrative Science Quarterly, 1995, 40(2):256-282.

[4] Sitkin S B, Weingart L R. Determinants of risky decision-making behavior: a test of the mediating role of risk perceptions and propensity. Academy of Management Journal, 1995, 38(6):1573-1592.

[5] 白云涛, 郭菊娥,席酉民.高层管理团队风 险偏好异质性对战略投资决策影响效应 的实验研究. 南开管理评论,2007, 10(2):25-30.

[6] Ring P S, Ven A H V D. Developmental processes of cooperative inter organizational relationships. Academy of Management Review,1994, 19(1):90-118.

[7] Simons T L, Peterson R S. Task conflict and relationship conflict in top management teams: The pivotal role of intragroup trust. Journal of Applied Psychology, 2000, 85(1):102.

[8] 毛华配，王艇，郑璐等. 投资者风险感知 与风险倾向对风险决策的影响——基于 民间投资者的样本. 应用心理学, 2013, 19(4):332-338.

[9] Sun Y Y, Yang Q S. A Study of spatial evolution patterns of tourist destinations disaster risks. Journal of Risk Analysis and Crisis Response, 2018, 8(1): 35-42.

[10] 胡桂兰. 创业团队异质性对创业决策的影 响研究.江苏大学, 2013.

[11] Yuan S X, Huang G Q, Xiong H X, Gong Q H, Wang J, Chen J. Maximum entropy-based model of high-threat landslide disaster distribution in Zhaoqing, China. Journal of Risk Analysis and Crisis Response, 2017, 7(3): 108-126

[12] 黄杰, 朱正威, 赵巍. 风险感知、应对策略与 冲突升级一一个群体性事件发生机理 的解释框架及运用.复旦学报(社会科学 版), 2015, 57(1):134-143.

[13] Williams D J, Noyes J M. How does our perception of risk influence decision- making? Implications for the design of risk information. Theoretical Issues in Ergonomics Science, 2007, 8(1):1-35.

[14] Ellis A P J. System Breakdown: The role of mental models and transactive memory in the relationship between acute stress and team performance. Academy of Management Journal, 2006, 49(3):576-589.

[15] 肖璐.高管团队信任对组织绩效的影响:团 队冲突的中介作用. 经济研究导 刊,2010(6):95-98.

[16] 张涛,刘延平,赖斌慧. 团队冲突和团队信 任对团队心智模式形成影响的实证研究. 北京交通大学学报(社会科学版), 2008, 07(4):58-63.

[17] 陈璐,杨百寅,井润田,等. 高层管理团队内 部社会资本、团队冲突和决策效果的关系 ——研究综述与理论分析框架. 南开管理 评论, 2009, 12(6):42-50.

[18] Langfred C W. Too Much of a Good Thing? Negative Effects of High Trust and Individual Autonomy in Self-Managing Teams. Academy of Management Journal, 2004, 47(3):385-399.

[19] Mackinnon D P, Fritz M S, Williams J, et al. Distribution of the product confidence limits for the indirect effect: Program PRODCLIN. Behavior Research Methods, 2007, 39(3):384-389. 